\title{
Entender a Rusia a través de sus fuerzas profundas: dificultades y desafíos de una reflexión recurrente
}

\author{
Understanding Russia trough its deep strengths: difficulties \\ and challenges of an eternal reflection
}

GRACIELA ZUBELZÚ

Rev. Bras. Polít. Int. 50 (1): 102-120 [2007]

\section{Introducción}

El concepto de Fuerzas Profundas ha sido acuñado por Pierre Renouvin y Jean Baptiste Duroselle en el estudio de la Historia de las Relaciones Internacionales con el objeto de aprehender el fenómeno internacional en todos sus aspectos. Este concepto aunque central no supone una relegación del rol del Estado, en cuanto único actor que conduce a la política exterior, política pública que manifiesta el comportamiento y refleja el posicionamiento internacional de esos actores. En consecuencia, los conceptos de Fuerzas Profundas y Estado resultan claves para analizar el peso de las primeras en los criterios centrales que guían a la política exterior del segundo.

Renouviny Duroselle afirman que "las condiciones geográficas, los movimientos demográficos, los intereses económicos y financieros, las características mentales colectivas, las grandes corrientes sentimentales, nos muestran las fuerzas profundas que han formado el marco de las relaciones entre grupos humanos y que, en gran medida han determinado su naturaleza" (Renouvin y Duroselle, 2000: 9, 10). Desde nuestra perspectiva el concepto de fuerzas profundas abarca y contiene aquellos elementos que denominamos constitutivos de la identidad, asignando a los mismos un valor primario.

Un estudio anterior se centró en la cuestión del nacionalismo como una de las fuerzas profundas en la Rusia contemporánea (Zubelzú, 2005). Esta fuerza resultó un elemento aglutinador a la hora de la reconstrucción identitaria de una Rusia que experimenta, a partir de 1991, por primera vez en su historia, una novedosa condición de Estado-nación. De ahí el análisis privilegiado que se le otorgó.

En este artículo se pretende identifica un conjunto más amplio de rasgos que por su persistencia en prolongados períodos históricos pueden ser considerados

\footnotetext{
* Doctora en Relaciones Internacionales. Investigadora del Consejo Nacional de Investigaciones Científicas y Técnicas (CONICET). Docente en la Universidad Nacional de Rosario - Argentina (gzubelzu@unr.edu.ar).
} 
como elementos que también contribuyen a moldear la identidad de la nación rusa. En consonancia con el enfoque de Lafer quien sostiene - al analizar el caso de Brasil - que estos factores de persistencia son los que ayudan a explicar rasgos importantes de la identidad de un país (Lafer, 2001: 25). La condición de la persistencia, aunque esta sea cíclica en su intensidad, es un punto a destacar en particular al abordar el caso ruso.

Otro concepto necesario que complementa al de fuerzas profundas es el de fuerzas organizadas entendiendo por tales a agencias, actores estatales y burocracias. Las relaciones recíprocas entre ambas fuerzas constituyen el punto esencial de cualquier estudio político interno, pero también, de cualquier estudio sobre relaciones internacionales. En muchos casos, las fuerzas organizadas pueden operar modelando o suavizando las fuerzas profundas, pero también en otros casos, las "fuerzas organizadas" rescatan o realzan esas fuerzas profundas, tales son los casos del nacionalismo y la relevancia del espacio, entre otros (Colacrai; Lorenzini, 2005).

La impronta de las fuerzas profundas, aunque con variada intensidad según el factor temporal, permearía el elemento organizativo y tendería a impregnarlo. En el caso ruso, por ejemplo, numerosos autores señalan que sin la natural o ancestral adhesión del campesinado ruso a la vida comunitaria el socialismo cuyo punto de partida teórico era el individuo alienado y proletarizado- no podría haberse desarrollado en el país.

El punto de partida de este artículo, como en el estudio anterior donde se abordó el nacionalismo, también está dado por la contemporaneidad del debate acerca de la construcción identitaria de Rusia como una nación -además de un Estado-nacióndespegada por primera vez, al menos territorialmente, de su componente imperial. Ese despegue o desacople formalizado con las independencias de los Estados possoviéticos en 1991, constituye un proceso aún inconcluso ${ }^{1}$.

Puede afirmarse que en gran medida el actual debate acerca de la identidad rusa se hace más complejo al enmarcarse en el contexto más amplio de la pos guerra fría. Esta se caracteriza por tendencias "centrífugas" (Gaddis; 1991) como la fragmentación de Estados, las migraciones, las luchas étnicas y religiosas intraestatales. El debate identitario también adquiere otra dimensión novedosa para el caso de la Rusia pos-soviética que lo proyecta fuera de sus fronteras al estar inmersa Rusia y todo lo que en ella acontece, en un mundo de comunicaciones de alcance global que la ha integrado a él. Es en este marco en el que la nación rusa define no sólo "qué nos une" sino también "qué nos separa" en sus relaciones con "el otro" en un contexto internacional de comunicaciones simultáneas y extendidas.

1 Para un análisis de la década del 90 acerca de la política exterior rusa y el abandono de su rol de potencia imperial puede verse ARON, Leon, "The Foreign Policy Doctrine of Postcommunist Russia and its Domestic Context", en MANDELBAUM, Michael Ed., The New Russian Foreign Policy, Council on Foreign Relations, USA, 1998, pp. 23-63. 
Una primera aproximación a la cuestión indicaba que algunas fuerzas profundas están presentes, sea de modo abierto y visible, o más bien soterradas. La gran mayoría de ellas persisten o se desarrollan como fuerzas contrapuestas que conviven en tensión, más que fuerzas que logran un claro predominio. Ello conduce a que el análisis retome los debates que esa contraposición genera en algunos casos. La caracterización de estas fuerzas que a continuación se desarrollan, tiene por objeto mostrar cómo su presencia y vigencia subyace e influye en la política exterior. Dado que es precisamente la política externa rusa el foco último de atención de este artículo, la relación con "el otro" se torna central. En consecuencia, se procurará mostrar la percepción del otro (no cualquier "otro" sino el que sirve de referencia respecto a Rusia.

\section{El excepcionalismo y el mesianismo}

Entre el conjunto de fuerzas persistentes, la idea de excepcionalismo ha estado y permanece presente en la identidad nacional y en el discurso político de los Estados que han desempeñado el rol de actores centrales (Imperios, Grandes Potencias) en el sistema internacional. Aunque las razones del excepcionalismo que los Estados se auto adjudican varíen, así como también su uso político, el concepto se relaciona con otros como el mesianismo, el externalismo ${ }^{2}$. Ambos conceptos comparten la idea de contar con un acervo o patrimonio cualitativamente distinto y superior al de otros pueblos y países. Sin embargo, el segundo concepto incluye o supone una actitud más proactiva y una firme convicción en la necesidad de actuar en pos de la conversión del "otro". Aquí se diferencia a estos términos entendiendo que el mesianismo tiene un componente religioso, en tanto el excepcionalismo se apoya en rasgos seculares y culturales.

El excepcionalismo ha ido variando de contenido según distintos períodos de la historia rusa. En algunos de ellos se basó en una superioridad moral, en otros en una misión espiritual respecto a otros pueblos menos afortunados. Hay quienes han señalado que a partir de la constitución de la URSS el internacionalismo destinado a proclamar la superioridad del modelo socialista, repitió pautas de conductas "misionales" bajo formas seculares que paradójicamente consideraban a la religión "el opio de los pueblos".

Richmond sostiene que el mesianismo está todavía vivo en la Rusia actual, especialmente entre los intelectuales, tanto de derecha como de izquierda, quienes comparten la creencia y el orgullo de Rusia como un gran poder con una especial misión en el mundo 3 .

2 Externalismo entendido como lo señalaba José Luis de Imaz en el sentido de colocar las responsabilidades políticas de situaciones negativas en el contexto externo en detrimento de profundizar el análisis acerca de responsabilidades y márgenes de acciones políticas destinadas a remover obstáculos internos.

3 RICHMOND, Yale, "Culture and Character", "Negotiating with Russians", From Nyet to Da: Understanding the Russians, (Interact Series), 2003, p. 61. 
La idea de superioridad y excepcionalismo que se evidencia en el reclamo de ser un pueblo elegido tiene profundas raíces en la cultura religiosa rusa. Desde la Edad Media la Iglesia Ortodoxa fue la única comunidad religiosa independiente, funcionando bajo una soberanía Ortodoxa más que bajo el gobierno extranjero Otomano como fue el caso con las otras iglesias ortodoxas. Esto fortaleció a la Iglesia Ortodoxa en Rusia y a su reclamo de ser la única portadora de la verdad revelada. Con la incorporación de la Iglesia en las instituciones estatales - las fuerzas organizadas - desde principios del siglo XVIII una autocomprensión teológica y un mesianismo político crecieron aún más estrechamente conectados ${ }^{4}$. Ello condujo a la nacionalización de la iglesia rusa y a la consolidación de la visión de Moscú como la Tercera Roma. Esta, a diferencia de la primera Roma y de la segunda Constantinopla no había caído en manos de herejes como los bárbaros y los turcos. En consecuencia desde la caída de Constantinopla, Moscú asumió el peso y la gloria de llevar consigo la verdad cristiana autopercibiéndose como un faro de la fe.

Así durante un prolongado período de la historia rusa, el componente mesiánico ha tenido contenido religioso. Sin embargo, hacia el siglo XIX los eslavófilos comenzaron a desarrollar formas seculares del mesianismo. Así, la aún joven, enérgica y omnicomprensiva cultura rusa fue contrastada con la cultura materialista, corrupta y racionalista de Europa, la cual tendría que volver hacia oriente para regenerarse ${ }^{5}$.

En el caso ruso la condición de la excepcionalidad se entronca con una barrera que los rusos imponen frente al 'no ruso' y que consiste en su pétrea convicción, experimentada por cualquiera que tenga trato con ellos, acerca de la imposibilidad de ser comprendidos por un 'no ruso'. En palabras de Lourie "Que los rusos eluden el poder ser entendidos o comprendidos -porque son demasiado diferentes, demasiados profundos o también irracionales- es una imagen promovida tanto por los rusos como por los extranjeros". Así el autor ofrece ejemplos: Winston Churchill definió a Rusia "como un acertijo envuelto en un misterio, adentro de un enigma"; Fyodor Tyutchev, el poeta y diplomático eslavófilo del siglo XIX, escribió que "la esencia rusa era invisible a los ojos arrogantes de los extranjeros" y el filósofo del siglo XX Nikolai Berdayev fue tan lejos que dijo que "la psiquis rusa está estructurada de forma diferente de la Europea, los rusos tienen el inconsciente donde los europeos tienen el consiente y vice-versa" 6 .

La apertura realizada en la URSS a partir de la glasnot desde mediados de los años 80 , posibilitó la reflexión pública sobre el pensamiento de filósofos como el

4 SIMON, Gerhard, "The Historical Prerequisites for Russia’s Modernisation", Aussenpolitik 1/98, p. $67-84$, p. 76.

5 Ibidem.

6 LOURIE, Richard, "Reading the Russians", The Moscow Times, July 10, 2006. Lourie es el autor de "The Autobiography of Joseph Stalin" y "Sakharov: A Biography." 
ya citado Beryaev quien sostenía que "el destino de Rusia era el de ser una nueva Jerusalem y que los rusos eran un pueblo orgánico especialmente espiritual con una misión de transformar la sociedad". También se discutieron las ideas de Vladimir Solovyov, un filósofo del siglo XIX cuyas obras completas fueron publicadas por primera vez hacia fines de la década del 90. Solovyov acuñó el término "la idea rusa", que contenía una mística muy determinada y veía a la cristiandad como el repositorio de la suprema sabiduría. Estas ideas que replanteaban otras similares de mayor antigüedad fueron silenciadas para ocultar su vigencia o supervivencia contemporánea a la atea "ideología oficial". La fragmentación de la URSS y el abandono de esta ideología dejaron el terreno libre al debate y adopción de nuevas cosmovisiones. El retorno o la adopción de "la idea rusa" ha preocupado a quienes temen que la creencia en el excepcionalismo ruso pueda transformarse en un mesianismo peligroso ${ }^{7}$.

En su obra sobre los rasgos culturales de los rusos y de Rusia, que contiene reflexiones comparativas con la cultura americana, Richmond Yale al analizar el mesianismo ruso lo considera un rasgo de contacto entre ambas. "Tales misiones mesiánicas no son desconocidas por los americanos, quienes en diversos momentos históricos han creído que cuentan con algo especial para llevarles a los menos afortunados: Cristiandad a los bárbaros, democracias a los gobernados por dictadores y el libre mercado a los economías dirigidas por el Estado"8. Como puede observarse Yale incluye componentes seculares en su empleo del término mesianismo a diferencia de las precisiones conceptuales que consideramos convenientes señalar en párrafos anteriores.

\section{El externalismo}

Existe también una tendencia a ubicar la causa de los problemas del país en actores foráneos, en "el otro". Así, el externalismo se complementa de modo lógico con el excepcionalismo. En el caso que aquí se aborda si los rusos son el pueblo elegido y tienen el monopolio de la verdad, de la fe religiosa o de la superioridad moral, en momentos de dificultades, guerras, decadencia, "otros" deben ser la causa de sus desgracias. Los masones y los judíos en el pasado han sido culpados con frecuencia por los problemas rusos. En tiempos recientes se los ha acusado por las dificultades económicas del país ${ }^{9}$. Aunque manifestaciones de este tipo encienden rápidamente alarmas, y es bueno que así sea, puede afirmarse que el antisemitismo resulta un fenómeno marginal no alentado por el poder político ${ }^{10}$.

\footnotetext{
7 COHEN, Patricia, "Freed From Ideology, Russian Philosophers Explore Limitless Possibilities”, New York Times, March 13, 1999.

8 RICHMOND, Op.Cit., p. 62.

9 Ibidem, p. 63.

10 Ibidem.
} 
La visión de Rusia como víctima, y en consecuencia el externalismo, se acentuó enfáticamente desde la iniciación del proceso de reformas de Yeltsin, que dejó como saldo un profundo deterioro en las condiciones de vida de la mayor parte de la población. Esta vez Rusia sufría a manos de las fuerzas malignas de Occidente, enemigo (siempre referente) ancestral de la cultura ortodoxa. El cambio de victimario -el sistema soviético por los países desarrollados de Occidente- se hace en paralelo al debilitamiento del Estado soviético y su desaparición. Así la fuerza del externalismo explica la alianza que entonces se produjo entre los nacionalistas mesiánicos y los comunistas, evidenciada de modo claro en el Congreso de los Diputados del Pueblo Ruso y en el Soviet Supremo de la todavía Unión Soviética.

El externalismo constituye un elemento palpable en el análisis y la retórica contemporánea del que se citan algunos ejemplos. Así Alexei Pushkov, quien supo escribir discursos para Gorbachov, sostiene que "Rusia puede fragmentarse si falla en reestablecer su papel de gran potencia económica y militar". En la actualidad en un programa televisivo suele responsabilizar regularmente a los enemigos de Rusia - los americanos, los ciudadanos de los estados Bálticos, los polacos y los georgianos - de las desgracias del país en los últimos 15 años ${ }^{11}$.

Para entender esta percepción de "fortaleza asediada" común a las élites y a la nación rusa, nada mejor que el aporte del periodista americano William Pfaff que trabajó desde los medios atacando a la entonces URSS. Su análisis en un artículo cuyo título - Why make an enemy of Russia? - representa una toma de posición e ilumina sobre la visión de los propios rusos. Para responder a su autopregunta primero señala las diferencias fundamentales entre la entonces URSS y la Rusia actual. A la primera la consideraba un país poderoso, hostil y déspota que dominaba a Europa Central y Oriental contra la voluntad de sus poblaciones, mientras que Rusia es un socio estratégico de Occidente. En el plano interno "Putin puede controlar la televisión nacional, pero la prensa y la discusión pública son libres. Los rusos incuestionablemente lo respaldan y hay controversias y un vigoroso debate político y elecciones periódicas". Y respecto a la política exterior, Pfaff afirma que Moscú coopera con Occidente en prácticamente todos los niveles de las relaciones internacionales: lo abastece con petróleo, coopera en la guerra antiterrorista de Bush y no cuestionó el establecimiento de bases norteamericanas en Asia Central. Después de esta evaluación reformula la pregunta en términos de la política norteamericana ("So why do we want to make an enemy of Putin?") y plantea una respuesta cuyos argumentos pueden ser transferidos para representar la lógica rusa. Así Pfaff entiende que "los rusos están siendo sujetos de un muy alto nivel de provocación. Rusia está ahora rodeada por el poder americano. Hay fuerzas estadounidenses en Asia Central y en el Cáucaso. Con los estados Bálticos

11 PFAFF, William, "Why make an enemy of Russia?", International Herald Tribune, Wednesday, April 13, 2005. 
como miembros de la OTAN, la aviación de la alianza está desplegada en la frontera rusa. Los polacos y otros están ansiosos de que Ucrania se una a la OTAN y a la UE. El gobierno ruso se ha mantenido sorprendentemente calmo ante todo esto, pero uno de estos días puede perder la calma. Rusia hoy no es la Unión Soviética, pero puede encontrar aún modos de ser muy desagradable para quienes eligen hacer de ella un enemigo ${ }^{12}$."

A modo de complemento de las visiones anteriores se considera imprescindible incluir la percepción sobre el externalismo y su relación con los medios de comunicación. Para tratar de testear cuán acentuada es esta tendencia actualmente en los medios de comunicación rusos, la periodista Olga Romanova, hizo la prueba de pasar tres días sin navegar por Internet y sin mirar por TV satelital canales occidentales. Para mostrar el nivel de impacto alcanzado por los medios, Romanova sostuvo que "después de ese lapso entendí que Rusia está rodeada de enemigos y que Putin es nuestra gran esperanza"13. Aunque no es el propósito aquí analizar el rol e impacto de los medios en la construcción identitaria y en la influencia sobre la opinión pública y el electorado, interesa al menos señalar que la visión de Rusia como víctima no es un dato irrelevante ${ }^{14}$. Más allá de si se estimula o no esta visión desde el Estado, lo cierto es que existe una propensión en la sociedad a responsabilizar a terceros de los problemas del país y que tal externalismo puede palparse en los medios de comunicación.

\section{La extensión territorial: un país, dos continentes}

La dimensión continental de Rusia, el país más extenso del planeta ${ }^{15}$, y la vulnerabilidad/o desafío que el control efectivo de ese gigantesco territorio supone, han constituido condicionantes permanentes que contribuyeron a moldear una de las fuerzas profundas de la identidad rusa: el respaldo a una autoridad concentrada $y$ fuerte. Aún en la actualidad, y bajo las nuevas realidades del mundo de posguerra fría, el condicionante territorial, en combinación con otros factores revela su enorme peso. Algunos datos bastarán para resaltar la dimensión de la cuestión: Rusia tiene 11 husos horarios (EEUU tiene seis); un viaje desde Moscú hasta Vladivostok, sobre el Pacífico, en el tren transiberiano que se extiende por más de un tercio de la circunferencia terrestre, insumirá 7 días; Rusia tiene actualmente fronteras con 14 países (15 si se incluye a Mongolia).

12 Ibidem. Pfaff analista del Internacional Herald Tribune trabajó en la organización Europa Libre pionera en transmiciones y, como el mismo dice en el artículo, otras formas de combate dirigidas directamente contra los regímenes comunistas de Europa Central y Oriental.

13 MAYR, Walter; NEEF, Christian, "Russia’s Return. A Former Superpower Rises Again", Der Spiegel (Germany), July 10, 2006.

14 Según cifras oficiales Rusia contaba en el 2006 con 25 millones de usuarios de Internet. MOSCOW, December 18 (Itar-Tass) - Russian Information Technologies and Communication Minister Leonid Reiman.

15 Un octavo de la superficie terrestre total. 
El respaldo a una autoridad con escasos límites se ha consolidado por otros factores (memoria histórica de invasiones del sur, no existencia de barreras geográficas, aislamiento que fortaleció la cristalización de modos de organizar la autoridad política y la organización social y que a su vez neutralizó la innovación). Es importante destacar que autores como Foglesong y Han refutan mitos tanto destinados a alimentar el excepcionalismo ruso como aquellos otros destinados a considerar a Rusia como un país común o "sujeto a las leyes universales del desarrollo" (mito 3). Sin embargo, respecto a este último destacan que el factor geopolítico es un dato relevante a la hora de considerar la conducta actual del Estado ruso. Así señalan, por un lado, el enorme costo que el condicionante territorial supone en infraestructura y en defensa, así como los efectos políticos de ser el único país en calidad de vecino de mundos en turbulencia (los países musulmanes), cambio (la Europa ampliada) y ebullición (asiáticos) ${ }^{16}$. Las características particulares de esta extensión territorial continental han actuado en la historia ruso/soviética como un factor determinante a la hora de moldear sus instituciones políticas. Aunque los cambios tecnológicos - en transporte, comunicaciones y seguridad - han ido reduciendo las dificultades que esto supone, la persistencia o la profundización de problemas como el crecimiento demográfico negativo ruso acentuado en algunas regiones del Lejano Oriente siguen demostrando la importancia de este condicionante.

\section{La concentración de la autoridad política: el peso del Estado}

El Estado ha jugado un rol de enorme peso en la vida rusa. Esta tradición ha atravesado diversos sistemas políticos como el imperial-autocrático y el soviético. Numerosos son los análisis que se focalizan en la magnitud y alcance de su influencia actual en relación a otras categorías claves del pensamiento político occidental: la sociedad y el mercado. En el primer caso sabemos que transformaciones en la relación estado-sociedad civil requieren períodos prolongados de tiempo para sedimentar, más allá de los cambios o de la acción de las "fuerzas organizadas". También conocemos que esas transformaciones ocurren de modo no lineal y adoptan formas propias más que una conversión a moldes o modelos ajenos. En el segundo caso que refiere al vínculo Estado-mercado debemos precisar que aunque se considere en términos muy generales al mercado como el ámbito del libre juego de la oferta y la demanda, acá nos focalizaremos en un aspecto particular de esta idea abarcativa: el sector privado como propietario de bienes frente al sector público.

Son dos entonce los aspectos a considerar respecto al peso de Estado en la vida rusa. Surgen así dos preguntas centrales: ¿Cuál es o será la combinación óptima de Estado y sector privado en el capitalismo ruso? Y ¿cuál será el grado de poder que

16 FOGLESONG, David; HAHN, Gordon, “Ten Myths About Russia. Understanding and Dealing with Russias Complexity and Ambiguity", Problems of Post-Communism, N. 49, November/December 2002, pp. 3-15. 
debe tener el Estado en relación a los otros poderes republicanos constituidos, el legislativo, el judicial, los poderes de los miembros de la federación, y también la sociedad civil?

En torno a estas preguntas, más que brindar respuestas claras, se busca profundizar la reflexión. Como punto de partida puede tomarse una visión ampliamente aceptada de la sociedad rusa que puede ser interpretada con las siguientes palabras "el mercado es un lugar donde todo esta a la venta y donde todo puede ser comprado. Una economía de mercado inevitablemente conduce a una gran estatificación social y a una floreciente corrupción. Desde que los ciudadanos rusos fueron hipnotizados en "zoombies" con la idea del mercado, que es absolutamente extraña/extranjera para el espíritu ruso, nuestra sociedad se ha transformado en venal desde arriba hacia abajo" ${ }^{17}$.

Para complementar esto, y nuevamente tomando como referencia a Europa y las variantes europeas de capitalismo, cabe enmarcar este rechazo a la idea del Estado mínimo y del mercado omnipotente en el análisis que realiza el profesor Gorshkov cuándo se le pregunta cuál de los tres modelos -el liberal británico, el continental alemán o el sociodemócrata sueco - está más cerca de las expectativas de los ciudadanos rusos.

Gorshkov afirma que "en Rusia, donde el Estado juega un rol exagerado como sujeto principal de la política social, las características del modelo liberal son consideradas absolutamente inaceptables: (esto es) minimizar la intervención del Estado en la esfera social, una división estricta de responsabilidad para varios problemas sociales entre los diveros niveles de gobierno, y los ciudadanos ellos mismos tomando máximas responsabilidades por su propio bienestar Apoyándose en una encuesta señala que sólo un $5 \%$ de los entrevistados consideró que la gente debe resolver sus problemas por si mismos, y no confiar en el Estado. El grupo mayoritario (50\%) sostiene que el Estado debería proteger a todos los pobres. Ante la pregunta acerca de si esto demuestra la continuidad de un principio de la era socialista, Gorshkov plantea un dato muy interesante, al afirmar que el porcentaje de encuestados que cree que el Estado debe proteger a todos los pobres ha crecido drásticamente, se ha duplicado, en relación a la década pasada. A su vez, ha habido un caída abrupta, más del $60 \%$, en la proporción de los encuestados que creen que el Estado debe pagar únicamente beneficios a la gente que no es capaz de trabajar. También el porcentaje que vincula la solución de los problemas sociales con las empresas se ha reducido a un tercio de su nivel previo ${ }^{18}$. Estas y otras señales indican que dado el peso de las fuerzas profundas, en este caso

17 ANTONOV, Mikhail, "Does Putin need a third term? Vladimir Putin: a strong ruler for a great power", Literaturnaya Gazeta, No. 47 November 24, 2006, Translated by Elena Leonova

18 Novaya Gazeta, "The State is for Me", No. 53, July 17-19, 2006. Entrevista con el sociólogo Mikhail Gorshkov realizada por Irina Timofeeva. Esta entrevista se enmarca en un estudio hecho por el Instituto de Sociología de la Academia de Ciencias de Rusia y la Fundación Friedrich Ebert de Alemania. El informe de Timofeeva titulado "Social Policy and Social Reforms as Perceived by RussianCitizens," que se focaliza en qué clase de modelo socio-político los rusos consideran óptimo fue publicado en junio de 2006. 
el aval al rol del Estado en relación al Mercado, puede plantearse un proceso de transformación que asemeje el vínculo de esa dupla con el del capitalismo escandinavo. Se entiende que solo un proceso gradual que opere más adoptando cambios cuyos referentes se ubiquen en el extremo del continum más cercano a las realidades presentes e históricas de las sociedades puede lograr afirmarse. La variante liberal del capitalismo ensayada en Rusia a principios de los 90' no ha sido exitosa y ha llevado a su revisión y desprestigio. Numerosos analistas e historiadores han remarcado que subsiste en la población rusa una especie de ética del igualitarismo. Como señala Richmond el igualitarismo debe ser entendido como la creencia en que las condiciones materiales de vida en la sociedad no deben tener variaciones muy marcadas entre los individuos y las clases. El igualitarismo constituye una filosofía social que defiende la equidad entre las personas y una más equilibrada distribución de los beneficios. Así, la ideología comunista se apoyó en una convicción arraigada en las tradiciones de la comunidad rural $(\mathrm{mir})^{19}$. De ahí puede entenderse el profundo desprecio que la mayor parte de los rusos tienen por los llamados "nuevos rusos" (novii ruskii) el grupo de hombres de negocios que hizo su fortuna a principios de los 90'. Ese mismo rechazo constituye una de las razones de apoyo al presidente Putin en relación a su campaña contra los novii ruskii "quienes saquearon al Estado y a Rusia".

Otro aspecto de la reflexión en relación al estatismo, entendido como preeminencia de uno de los otros poderes republicanos constituidos - el ejecutivo - por sobre el legislativo, el judicial, los poderes de los miembros de la federación, y también por sobre la sociedad. La concentración del poder político en Rusia constituye un hecho histórico pero también una fuerza profunda. Numerosos pensadores y analistas coinciden en señalar que las características del país requieren un gobierno fuerte. En palabras de Antonov por ejemplo: "régimen estricto, no necesariamente brutal", "fuerte pero justo". Los períodos históricos en los que la autoridad estatal prácticamente se desplomó - el más reciente, principios de los 90' - son percibidos por los rusos con una enorme aprehensión y rechazo. La reconstitución o establecimiento de la autoridad que evite el caos y el desorden resulta una prioridad a la cual se relegan claramente otras metas. Como sostenía Bull el orden es el prerrequisito indispensable para la obtención de otros bienes sociales.

A partir de esta consolidada visión para Simon "una especie de omniresponsabilidad es asignada al Estado: no solo por el bienestar de todos, por la seguridad social, por la garantía del empleo su salud y educación sino también por darle significado a todo. Existe un difundido deseo de una nueva ideología nacional para ser implementada por los órganos del Estado." Podemos afirmar que en esta visión las fuerzas organizadas interpretarían las demandas surgidas de las fuerzas profundas.

19 RICHMOND, op. cit., p. 36. 
Por otro lado, y fortaleciendo la idea de la nación rusa como tierra de tensiones irresueltas o de contradicciones, cabe señalar el complejo vínculo del hombre común ruso con las fuerzas organizadas. Simon señala que el ruso desconfía profundamente de las instituciones - gobierno, parlamento, autoridades, la policía. Así, estudios sociológicos de opinión muestran que las instituciones han estado en los niveles más bajo de valoración por muchos años. La evasión impositiva, las coimas, la ignorancia o desobediencia de las instrucciones de la policía son aún consideradas como parte de las reglas del juego. Sus categóricas palabras definen que "El Estado Leviathan es considerado tan peligroso como poderoso, un estado del cual es habitual tomar todo lo que se puede y al cual es dado lo menos que sea posible" 20 .

Unos años después de que ese artículo fuera escrito el vice-primer ministro y ministro de Defensa - además de potencial sucesor de Putin - Sergei Ivanov, admitió que "en efecto, el índice de popularidad del Ejército de Rusia, igual que el de otras instituciones públicas, antaño era bastante bajo, pero ahora la situación va cambiando. A juzgar por los sondeos de opinión, el primer lugar según el índice de confianza lo ocupa la institución presidencial, a la que sigue la Iglesia y en el tercer lugar se encuentran las Fuerzas Armadas ${ }^{21}$.

\section{La eterna pregunta: ¿son los rusos europeos?} O ¿cuán europeos son los rusos?

La tensión entre el fortalecimiento de la identidad nacional y la pertenencia a la cultura europea ha sido otra tradición presentada predominantemente con carácter antagónico y planteando opciones excluyentes.

Sin embargo, resulta aquí de mayor interés señalar que esta visión adquiere más bien un carácter de tensión irresuelta presente en individuos y grupos y se manifiesta en diferentes ámbitos. Esta tensión devela una condición permanente: los rusos estaban y están inseguros de su lugar respecto a Europa y esa incertidumbre es una fuerza profunda de su historia y construcción identitaria. Esta tensión ha devenido de modo cíclico y dialéctico. Mientras la adhesión e imitación de lo europeo se inicia con la política de Pedro el Grande simbolizada en la construcción de la nueva capital imperial, San Petersburgo como la "ventana a Europa" (1703). La influencia del pensamiento y los escritos de Fonvizin de fines del siglo XVIII muy críticos de "Occidente" (entonces equivalente a Europa) por su decadencia moral, codicia material e importancia de lo superficial dejaron una marca profunda en prácticamente todos los escritores rusos desde Pushkin ${ }^{22}$.

20 SIMON, p. 72.

21 Boletín Informativo de la Embajada de la República Argentina en la Federación de Rusia, 30 de noviembre de 2006, no 139.

22 FIGES, Orlando, "El baile de Natacha. Una historia cultural rusa", Ediciones Edhasa, Madrid, 2006. traducción de Eduardo Hojean. capítulo sobre "La Rusia Europea”, p. 109. 
A partir de entonces el cuestionamiento y el rechazo surgieron de corrientes denominadas eslavófilas ${ }^{23}$.

Así la intelectualidad y las élites rusas recorren desde "la idealización de Europa" hasta "el rechazo", transformación que en gran medida se produce por el reino del terror que sucedió a la Revolución Francesa, donde el ideal de progreso e ilustración da paso a la violencia y a la destrucción.

Mientras desde los tiempos petrinos la presión a la adopción de pautas culturales europeas era además limitada a la nobleza de la Corte y a ciertos grupos sociales, el resto de la población el campesinado y los sectores de la iglesia se mostraban reacios a modificar costumbres, hábitos y valores propios. A partir de variadas raíces un grupo de intelectuales contrapuso una ideología que adquiere formas paneslávicas a mediados del siglo XIX y euroasiáticas a principios del XX.

El eurasianismo puede ser definido como un movimiento político e ideológico, que tiene sus raíces en la eslavofilia, sobre todo en la obra de Danilievski y Leontiev, quienes habían formulado la oposición religiosa y cultural, entre Rusia, Europa y Occidente. No obstante, constituye una ideología original dentro de la historia intelectual rusa e insiste en que Rusia - un país semiasiático y semi europeo, o más bien un continente aparte - debe inspirarse ante todo en el Oriente (Bizancio, Mongolia, China y Corea) ${ }^{24}$. Esta ideología asume que Rusia tiene un rol único en su historia, determinada por su tamaño, su identidad geográfica repartida en Europa y Asia, sus vínculos con el mundo musulmán y hasta su clima. Ellos creen que Rusia no es capaz ni desea adoptar los modos de vida occidentales ${ }^{25}$. En el plano político el ex primer ministro Yevgueny Primakov sería el representante más destacado de esta posición. Para Shlapentokh en la actualidad la mirada de un sector de la prensa escrita puede ser descripta como una versión de la idelogía Eurasiática.

Sin embargo, la corriente euroasiática no constituiría la corriente principal en términos identitarios y de opciones políticas. Aunque refleja el pensamiento de un sector importante no resulta la tendencia predominante.

En consecuencia, se destaca la reflexión de Figes, quien citando a Herzen ejemplifica "Necesitamos a Europa como un ideal, un reproche, un ejemplo escribió Herzen - si no fuera todas esas cosas habríamos tenido que inventarla". "La persistencia de estereotipos culturales (negativos) ilustra las proporciones míticas de "Europa" en la conciencia rusa. Aquella "Europa" imaginaria tenía más que ver con la necesidad de definir "Rusia" que con Occidente en sí mismo. La

23 La eslavofilia rusa fue una ideología conservadora que desarrollada en los años 40 del siglo XIX, que cultivó las fuentes nativas y eslavas de Rusia, y criticando la europeización del país, quiso regresar a los elementos auténticamente cristianos de la tradición social pre-pretina rusa.

24 Se puede ver RIZSANOVSKY, Nicholas V., "The Emergence of Eurasianism”, California Slavic Studies, 4, 1967, pp. 39-72.

25 SHLAPENTOKH, Vladimir, "Two Simplified Pictures of Putin's Russia, Both Wrong", World Policy Journal, Vol. XXII, Nro. 1, Spring 2005. 
idea de "Rusia" no podía existir sin "Occidente" (así como "Occidente" no podía existir sin "Oriente") 26.

Si bien la búsqueda de una identidad propia sufre el impacto de la revolución bolchevique y su complejo manejo y convivencia con el nacionalismo ruso, el debate identitario parece haber sobrevivido a esa etapa.

Así a partir de la apertura de una etapa de cambios en la década del 80', Europa vuelve a instalarse en el discurso oficial soviético. Gorbachov impulsó la idea de la "Casa Común Europea", destinada a mostrar las transformaciones internas y externas que buscaba producir en la URSS.

En el análisis realizado sobre el nacionalismo ruso (Zubelzú, 2005) se planteó en términos de las políticas a seguir a partir de 1991 la necesidad de establecer simultáneamente: 1) las fronteras del "nosotros" más general (russkoye o eslavos orientales) vínculo complejo pero existente entre rusos, bielorusos y ucranianos; 2) la necesidad política de un Estado que internamente estimule y fortalezca la inclusión de toda su población actual (rossianie); 3) y en relación a la diáspora rusa (russkii) aplique una política cauta y de cooperación en especial en las repúblicas pos-soviéticas.

Este complejo proceso de construcción del "nosotros" (russkoye-rossianierusskii) se desarrolla a la par que la relación con "el otro"27. Allí la referencia es Occidente, entendido como una entidad cultural que comprende a la civilización europeo-norteamericana, respecto a la cual Rusia mantiene un complejo y contradictorio vínculo. Occidente ha resultado tradicionalmente "el otro" para Rusia y ha constituido un elemento relevante en la conformación de su identidad. Este proceso puede adoptar dos tipos de estrategias y prácticas: las afirmativas, que remarcan las características positivas y celebran lo comunitario, y las negativas, que enfatizan las diferencias y la exclusión ${ }^{28}$. El empleo de estos mecanismos en la construcción identitaria cobran central relevancia a la hora de analizar las variantes del nacionalismo ruso. A su vez esta construcción puede poner el énfasis en elementos orgánicos o culturales o en elementos inorgánicos o ideológicos, entre los cuales sobresale la importancia de la dimensión externa. En su análisis del proceso identitario ruso, Kasianova subraya que en los años inmediatos a la fragmentación de la URSS, la élite rusa impulsó una autoidentificación con Occidente como comunidad de referencia, pero que ello requería un consenso de aquella que nunca se dio. Este rechazo, más intuitivo que consciente, llevó a las élites rusas a valorar el elemento orgánico de la identidad y a reforzar su peso en el modelo identitario. Este proceso no es novedoso, la historia rusa revela que estas

\footnotetext{
26 Op. cit., p. 110.

27 Resulta claro que aunque el russkii y el rossianie sea crecientemente el "nosotros", el resto de los eslavos orientales -ucranios y bielorrusos- no pasan a ser el grupo de referencia autoidentitaria: "el otro", sino que ellos mismos están involucrados en sus propios procesos de construcción identitaria.

28 KASIANOVA, Alla, "Russia in Search for the State Identity: the rol of the West", Tomsk State University, Russia Federation. (Unpublished Lecture), 2001.
} 
fuerzas profundas contrapuestas se acentuaron a fines del siglo XVIII y durante el siglo XIX se fortalece la identidad nacional tanto empleando prácticas afirmativas como negativas.

Esta tensión irresuelta refluye una y otra vez, condicionados por su ubicación y extensión geográfica, los rusos al vivir en las márgenes del continente europeo, jamás han estado completamente seguros de si su destino se encuentra realmente alli ${ }^{29}$. Y así resurge en términos antitéticos la eterna pregunta: ¿¿Son de Occidente o de Oriente? En esta pregunta subyace veladamente otra idea profunda, si no se es europeo en términos identitarios, Rusia mantendrá una posición de aislacionismo en el escenario internacional. Cabe aquí algunas aclaraciones, en primer lugar si se niega/o rechaza la pertenencia europea, la alternativa de ser orientales define en ese caso la propia distancia con la identidad europea, pero no una identidad que los acerque y reúna con Oriente. Es decir, se es oriental en relación a Europa pero esto no conlleva la inclusión (o la reivindicación de la inclusión) en Oriente, ni la construcción de una identidad que pivotee sobre ese eje, no al menos desde el poder político. En segundo lugar, en términos de política internacional la no pertenencia europea implicaba un distanciamiento de las potencias europeas con las que Rusia-URSS siempre demarcó alianzas (Santa Alianza, la Alemania nazi) y enemigos (la Francia Napoleónica, Gran Bretaña a mediados del siglo XIX, la Alemania nazi) y en consecuencia períodos de repliegue y aislamiento de la política exterior rusa. En tercer lugar, el aislacionismo no se corresponde con la política exterior del país desde que la URSS ejercita su condición de miembro permanente en el Consejo de Seguridad y extiende su influencia por varias regiones del planeta. Rusia, Estado continuador de aquella, ha redoblado la apuesta para ampliar su rol tanto en ámbitos internacionales excluyentes (G-8) como en instituciones internacionales como la Organización Mundial del Comercio. En consecuencia, aunque el aislacionismo como tendencia no se corresponda con las líneas que han guiado la conducta soviético/rusa desde la década del 50, debe entenderse que por un lado existen posiciones o discursos minoritarios que aún hoy sostienen esta propuesta y que cuando se habla de aislacionismo, el contenido parece otro. Así debe entenderse que se alude al plantear la imposibilidad o conveniencia de contar con aliados o socios estables o permanentes, más que proponer la disminución de los contactos y relaciones externas. Dada la densidad de los vínculos ruso-europeos en muchos casos también la referencia a Europa aparece cuando se habla de "una mayor aislamiento" ruso. Allí los obstáculos o circunstancias que perturban o dificultan estas relaciones bilaterales, suelen ser caracterizadas o entendidas como perspectivas de aislamiento ruso.

En contraposición con lo sostenido en los párrafos siguientes, el aislacionismo en algunas miradas resultaría una fuerza que persiste con intensidad. En esa línea el filósofo Isaiah Berlin diferenció el concepto de "isolate" del de "insulate". Entendía

29 FIGES, Ibidem. 
al primero como la descripción de la sección de la opinión pública americana que desea desasociarse del mundo externo, mientras sostenía que ese no era el caso de la actitud rusa. Rusia estaba preparada para participar de las relaciones internacionales, pero prefería que otros países se abstengan de interesarse en sus asuntos, es decir insulate a si misma del resto del mundo sin permanecer aislada de él ${ }^{30}$. El término insulate que no encuentra una traducción del inglés diferente de isolate, ya que en ambos casos empleamos aislar, connota en nuestro criterio una arraigada voluntad rusa por evitar la injerencia en sus asuntos internos, actitud que se ha plasmado de modo consecuente en la defensa de ese principio. Un estudio actual del prestigioso Centro Levada de Moscú avalaría la vigencia de tendencias como el aislamiento y la xenofobia. Así el número de aquellos que acuerdan con el slogan "Rusia para los rusos" ha aumentado de un $43 \%$ en 1998 a un $58 \%$ recientemente en un país que es el hogar de 160 pueblos y étnias ${ }^{31}$.

\section{La fuerza profunda en el plano político actual: la mirada europea}

Al explorar la relación entre Rusia y la UE el ex embajador británico en Moscú, Roderic Lyne vuelve a la eterna pregunta ¿Son los rusos europeos? Y a su respuesta afirmativa le siguen otros interrogantes ¿No son los rusos famosamente nacionalistas? ¿Orgullosos de su identidad e historia separada? ¿Puede decirse que tienen una aspiración europea? La existencia de una tendencia nacionalista no es patrimonio exclusivo ruso y el autor cita la dolorosa conversión de otros imperios como Gran Bretaña y Francia que han tenido que adaptarse a un cambio de status. Es claro que para el caso ruso los vínculos con la UE deben ser prioritarios: son los preferidos por la población según las encuestas, las otras opciones no son realistas, dado que por ejemplo China es más un motivo de preocupación estratégica de largo plazo.

En el balance respecto a la vinculación de tipo institucional si bien Lyne sostiene que "Actualmente es extremadamente difícil concebir a Rusia como miembro de la UE. Ni siquiera debe perderse tiempo en debatirlo. Rusia no solo es inconvenientemente extensa, sino que está muy pero muy lejos de alcanzar los requisitos que surgen de los principios de Copenhage. Los rusos no podrían completar el acquis communaitere de la UE. Ni desearían aceptar los aspectos supranacionales de la UE"32. Más aún, el diplomático afirma que el gobierno ruso no está ni siquiera cerca de preguntar acerca de la posibilidad de dar el primer paso en el largo camino hacia la membresía. Sin embargo, su estimulante reflexión sostiene que aunque esta posibilidad hoy este lejos no tiene que hacerles decir a algunos líderes europeos que jamás podrá darse. En definitiva plantea que

30 http://berlin.wolf.ox.ac.uk/lists/nachlass/whysovunfull.pdf (acceso 10 de diciembre de 2006).

31 MAYR, Walter; NEEF, Christian, "Russia’s Return. A Former Superpower Rises Again”, Der Spiegel (Germany), July 10, 2006.

32 LYNE, Roderic, "Russia in the EU?. We should never say never.", Europe’s World, Spring 2006, p. 38. 
respecto a los vínculos ruso-europeos, "Debemos cambiar modos de pensar por conceptos escleróticos dictados por circunstancias pasadas. E insistir en que Rusia debe aceptar las reglas de un club al que nunca le estará permitido ingresar no suena como una política con altas chances de ser exitosa".

Además el autor reconoce como elementos positivos:

En su último mensaje a la Asamblea Federal Putin habló de la adhesión de su país a los valores europeos. "Alcanzadas por la cultura europea a través de mucho sufrimiento, los ideales de libertad, derechos humanos, justicia y democracia han sido por muchas centurias los valores determinantes de nuestra sociedad". Aunque algunos críticos duden de la promoción de éstos es importante marcar que los valores sostenidos por el presidente no son distintos de los nuestros, como en el pasado ${ }^{33}$;

Los contactos con Europa que privilegian los rusos a la hora de los negocios, los estudios, el turismo e incluso a la hora de contar con viviendas o propiedades en el extranjero;

La legislación rusa ha tomado normas de la UE. Es probable que esta tendencia se fortalezca en los próximos 15-20 años, cuando la primer generación de líderes y gerentes pos soviéticos (muchos de los cuales han estudiado en Occidente o trabajado en o con empresas occidentales) pasen a estar en las primeras líneas de decisión ${ }^{34}$.

\section{La fuerza profunda en el plano político actual: la mirada rusa}

El presidente Putin definió al país como un país europeo y la ha hecho tanto en Rusia como en la propia Europa. En su mensaje de 2005 a la Asamblea Federal Putin habló de la adhesión de su país a los valores europeos. "Por sobre todo Rusia fue, es y por supuesto será una gran nación europea. Alcanzadas por la cultura europea a través de mucho sufrimiento, los ideales de libertad, derechos humanos, justicia y democracia han sido por muchas centurias los valores determinantes de nuestra sociedad" 35 .

En el exterior, en un muy citado discurso pronunciado en idioma alemán ante el Bundestag Putin remarca que Rusia es un país europeo y retoma la idea de un hogar común, señalando que "la cultura europea - a cuyo desarrollo Rusia ha contribuido - no conoce fronteras y cuya pertenencia siempre nos fue común y unió a nuestros pueblos." ${ }^{36}$. Resulta claro que dada la familiaridad con Alemania que el presidente ruso tiene, Berlín es el nexo europeo que facilita la conexión rusa.

En esta misma línea de recurrente indagación acerca de la pertenencia cultural europea de Rusia, las reflexiones del vice-jefe de la Administración

\footnotetext{
33 Op. cit., 40.

34 Ibidem.

35 Annual Adress to the Federal Assembly od the Russian Federation, April 25, 2005, The Kremlin. Moscow. www.kremlin.ru (Acceso 27 de abril de 2005)

36 "President Putin's address to the Bundestag", Berlin, September 25, 2001.
} 
Presidencial Vladimir Surkov resultan sumamente interesantes. "La versión rusa de la cultura europea es por supuesto una versión específica, pero no más específica que la alemana, francesa o británica ${ }^{37}$. Para intentar entender esa especificidad el funcionario aconseja leer a Dostoievsky quien rescata el rasgo contradictorio de la cultura rusa ${ }^{38}$.

A modo de balance entre la mirada europea y la rusa sobre la pertenencia identitaria rusa y su impacto sobre los vínculos futuros resulta interesante tomar una evaluación que resume con amplitud de miras el presente:

“En los próximos años Rusia será vista por muchos observadores como una sociedad peculiar que combina totalitarismo con algunas libertades individuales y feudalismo con una falta de habilidad para hacer cumplir sus propias leyes. Rusia sigue siendo una nación extremadamente heterogenea, no sólo social sino también territorialmente. Rusia posee una zona globalizada que incluye a Moscú y a San Petersburgo, a las provincias rusas y al Cáucaso no ruso. Cada uno de estos tres territorios pertenece a una época histórica diferente: el primero al siglo XXI (a la Rusia postindustrial), el segundo a los siglos XIX y XX (a la Rusia industrial) y el tercero a los tiempos de la Rusia patriarcal y pre industrial" 39 .

Entonces podría conjeturarse que la identidad europea de Rusia tiene, además de particularidades como tantas otras naciones según señalara Surkov, una concentración territorial que abarca con mayor intensidad al primer territorio, una menos palpable que comprende también al segundo y que no incorpora al tercero donde Oriente es más notorio. Esto no significa una segmentación identitaria de Rusia, sino todo lo contrario en esta coexistencia y heterogeneidad histórica parecería estar su originalidad.

\section{Reflexiones finales}

El interés que despierta el debate acerca de las posibilidades (e imposibilidades) de cambio en la Rusia actual en los ámbitos académicos y políticos no encuentra fácilmente parangón. Esta discusión involucra posiciones con alto contenido político, cuyas cosmovisiones están fuertemente arraigadas en la etapa de la Guerra Fría y en consecuencia hay poco de diálogo real o de apertura: o Rusia podrá ser democrática, moderna (pos-industrial) y una economía capitalista o Rusia seguirá siendo autoritaria, estatista y atrasada.

Este artículo ha buscado detectar con carácter preliminar y no excluyente las fuerzas profundas que permean la dinámica de la sociedad rusa. Algunas

37 LOURIE, op. cit.

38 Lourie realiza una lectura política de las palabras del funcionario, la que puede resumirse en "somos europeos pero tenemos nuestros rasgos propios, por ejemplo ser contradictorios y difíciles de entender para el no ruso, por eso no pueden criticar tan livianamente la concentración creciente del poder político".

39 Estas fueron las conclusiones de un debate sobre el futuro de Rusia en Literaturnaia Gazeta, December 14, 2004 citada por Shlapentokh, op. cit. 
de estas fuerzas, como el excepcionalismo, el mesianismo, el nacionalismo, son comunes a Estados que como Rusia han tenido un rol protagónico en el escenario internacional. En el proceso abierto a partir de la existencia de una Rusia no comunista resulta aún un interrogante el rol de la Iglesia Ortodoxa y de la religiosidad en el país. Si resulta claro que el actual gobierno apela a los valores representados por la fe ortodoxa y a un nacionalismo moderado con el propósito de ligar identitariamente a la nación rusa.

El condicionamiento y la preocupación territorial - positivo o negativo resultan factores de peso político permanente si se analizan períodos temporales extensos. El logro de la estabilidad de las fronteras alcanzado en distintos momentos históricos por diversos estados, constituye en el caso ruso un problema que ha comenzado a resolverse hacia fines de los 90'. Sin embargo, la extensión de sus fronteras y su tamaño sigue siendo un condicionante económico y político de perenne impacto en la política exterior y de seguridad.

El respaldo a la concentración de la autoridad política constituiría el caso más claro de convergencia entre la fuerza profunda con la fuerza organizada representada en un Estado de amplias competencias y capacidad de decisión.

La tensión entre el fortalecimiento de la identidad nacional rusa y la pertenencia a la cultura europea ha sido otro debate recurrente presentada predominantemente con carácter antagónico y planteando opciones excluyentes. Aquí resulta de mayor interés señalar que esta adquiere más bien un carácter de tensión irresuelta presente en individuos y grupos y se manifiesta en diferentes ámbitos y que muchas veces reacciona a la percepción del rechazo europeo. En el plano político, esa pertenencia constituye uno de los pilares en los que el gobierno apoya la prioridad de los vínculos bilaterales con Europa.

El rescate académico de estas fuerzas no apunta a considerarlas bajo un prisma determinista. Muy por el contrario el objetivo último consiste en destacar que las transformaciones perdurables ocurren - dialécticamente, cíclicamenteinvolucrando a las fuerzas profundas. El analista no puede ignorarlas pero tampoco entenderlas como condicionantes absolutos.

Recebido em 28 de março de 2007 Aprovado em 10 de junho de 2007

\section{Referências}

COLACRAI, Miryam \& LORENZINI, María Elena. "Identidad y Fuerzas Profundas en la Política Exterior de Chile", Titulo del Proyecto de Investigación: "Políticas Exteriores Comparadas: Primera Aproximación" (código 19/C137), Facultad de Ciencia Política y Relaciones Internacionales - Universidad Nacional de Rosario, 2005.

GADDIS, John Lewis. “Toward the post Cold War World”, Foreign Affairs, New York, Spring 1991, Vol. 70, Number 2. 
LAFER, Celso. La identidad Internacional de Brasil, Buenos Aires, Fondo de Cultura Económica, 2002.

RENOUVIN, Pierre \& DUROSSELLE, Jean-Baptiste. Introducción a la Historia de las Relaciones Internacionales, Fondo de Cultura Económica, 2000.

ZUBELZU, Graciela. "Rusia y la definición de sus intereses nacionales: la búsqueda de una guía en clave identitaria", Titulo del Proyecto de Investigación: "Politicas Exteriores Comparadas: Primera Aproximación" (código 19/C137), Facultad de Ciencia Política y Relaciones Internacionales - Universidad Nacional de Rosario, 2005.

\section{Resumen}

Este artículo analiza rasgos que por su persistencia en prolongados períodos históricos pueden ser considerados como elementos que contribuyen a moldear la identidad de la nación rusa. Estas "fuerzas profundas" son: el excepcionalismo, el mesianismo, el externalismo, el territorialismo y el peso del Estado. También se aborda a las "fuerzas organizadas" como actores estatales y burocracias. Se reflexiona sobre el peso y la vigencia de algunos de estas fuerzas en los debates sobre la política exterior contemporánea.

\section{Abstract}

This article analyzes features than, due to their persistence in extended historical periods, can be considered key elements to form the identity of the Russian Nation. These "deep forces" are: excepcionalism, messianism, externalism, territorialism and the key role of the State. The article deals with the "organized forces" such as burocracy and state agencies. It also considers the weigh and the standing of some of these streams in the debates over the contemporary foreign policy.

Palavras-chave: Rusia, Fuerzas profundas rusas, Mesianismo, Excepcionalismo, Estatismo. Key words: Russian deep strengths, Messianism, Excepcionalism, Statism. 